\title{
O processo de supervisão em ensino clínico. Perspectiva dos estudantes e enfermeiros'
}

\author{
Maria José Fonseca² \\ Salete Soares ${ }^{3}$ \\ Jacinta Gomes4 \\ Albertina Marques 5
}

doi: 10.11144/Javeriana.ie18-2.opse

Cómo citar: Fonseca MJ, Soares S, Gomes J, Marques A. O processo de supervisão em ensino clínico. Perspectiva dos estudantes e enfermeiros. Investig Enferm. Imagen Desarr. 2016;18(2): 77-88. http://dx.doi.org/10.11144/Javeriana.ie18-2.opse

1. Artigo original de pesquisa. Data de recebido: 20 de abril de 2015. Data de aceitação: 4 de decembro de 2015.

2. Mestre em Supervisão Portugal. Correio eletrônico: mjfonseca@ess.ipvc.pt

3. Doutora em Enfermagem Portugal. Correio eletrônico: saletesoares@ess.ipvc.pt Portugal.

4. Mestre em Enfermagem de Reabilitação Portugal. Correio eletrônico: jacintapisco@, gmail.com

5. Mestre em Enfermagem Portugal. Correio eletrônico: albertinamarques@ess.ipvc.pt 


\section{Resumo}

Introdução: O grande desafio que se coloca hoje à formação em enfermagem prende-se com o processo de (re) construção de saberes dos estudantes em ensino clínico onde a supervisão assume particular relevância. Objetivo: Conhecer a perceção dos enfermeiros tutores sobre a supervisão de estudantes de enfermagem em ensino clínico e conhecer a perceção dos estudantes de enfermagem sobre a supervisão em ensino clínico realizada pelos enfermeiros tutores, com a finalidade de contribuir com propostas para a praxis da supervisão. Metodo: Paradigma qualitativo, descritivo-exploratório. Participaram quinze estudantes de enfermagem e seis enfermeiros tutores, o instrumento de recolha de dados foi a entrevista semiestruturada, utilizando-se a análise de conteúdo segundo Bardin (1) no tratamento dos dados. Resultados: Os principais resultados revelam que os participantes concebem e percebem supervisão como processo de acompanhamento sistemático e orientação, potenciador do desenvolvimento de competências pessoais e profissionais, recorrendo a estratégias favorecedoras de reflexão. As dificuldades e necessidades dos enfermeiros tutores, relacionam-se com a formação em supervisão e maior disponibilidade para este processo. Os estudantes de enfermagem sugerem que os enfermeiros possuam conhecimento sobre alguns aspetos processuais de formação em ensino clínico e façam uma supervisão efetiva. As estratégias e conceção de supervisão destes participantes são coerentes com a finalidade preconizada para a supervisão de estudantes em ensino clinico, no entanto, emergem constrangimentos ao processo de supervisão. Conclusões: Parece-nos fundamental investir na formação em supervisão tal como numa gestão organizacional que possibilite a disponibilidade necessária para obter maior qualidade e segurança neste processo.

Palavras chave: estudantes; aprendizagem; supervisão; ensino clínico

\section{The Monitoring Process in Clinical Teaching. Perspective from Students and Nurses}

\section{Abstract}

Introduction: The challenge nursing education faces today is related to the process of (re)construction of knowledge by students in clinical training, where supervision is important. Objective: To know the perception of nurses tutors on the supervision of nursing students in clinical teaching and the perception of nursing students on clinical teaching supervision carried out by tutor nurses. Method: Qualitative, descriptive and exploratory paradigm. Fifteen nursing students and six tutor nurses participated. The data collection instrument was a semi - structured interview, with a subsequent content analysis according to Bardin. Results: It was revealed that participants conceive supervision as a process of systematic support and guidance, enhancing the development of personal and professional skills for those who resorted to strategies which favored reflection. The difficulties and needs of tutors nurses are training in supervision and greater availability for this process. Nursing students suggest that nurse tutors are aware of some procedural aspects of training in clinical teaching and make an effective supervision. Strategies and monitoring design of these participants are consistent with the purpose of supervising students in clinical teaching; however, limitations arise in the monitoring process. Conclusions: It seems essential to invest in training as an organizational procedure that allows the availability necessary to achieve a higher quality in the process.

Keywords: students; learning; supervision; clinical teaching 


\section{El proceso de supervisión en la enseñanza clínica. Perspectiva de los estudiantes y de los enfermeros}

\section{Resumen}

Introducción: El desafío que enfrenta hoy la educación de enfermería se relaciona con el proceso de (re)construcción de conocimientos de los estudiantes en la formación clínica, donde la supervisión es importante. Objetivo: conocer la percepción de los enfermeros tutores sobre la supervisión de estudiantes de enfermería en enseñanza clínica y la percepción de los estudiantes de enfermería sobre la supervisión en enseñanza clínica realizada por los enfermeros tutores. Método: Paradigma cualitativo, descriptivo-exploratorio. Participaron quince estudiantes de enfermería y seis enfermeros tutores. El instrumento de recolección de datos fue la entrevista semiestructurada, con análisis de contenido según Bardin en el tratamiento de los datos. Resultados: Se reveló que los participantes conciben la supervisión como proceso de acompañamiento sistemático y orientación, potenciador del desarrollo de aptitudes personales y profesionales, que recurrieron a estrategias favorecedoras de reflexión. Las dificultades y necesidades de los enfermeros tutores son la formación en supervisión y mayor disponibilidad para este proceso. Los estudiantes de enfermería sugieren que los enfermeros tutores tengan conocimiento de algunos aspectos de procedimiento de la formación en la enseñanza clínica y hacer una supervisión efectiva. Las estrategias y el diseño de la supervisión de estos participantes son consistentes con la finalidad de la supervisión de los estudiantes en la enseñanza clínica; sin embargo, surgen limitaciones en el proceso de supervisión. Conclusiones: Parece esencial invertir en la formación como una gestión organizativa que permite la disponibilidad necesaria para lograr una mayor calidad en el proceso.

Palabras clave: estudiantes; aprendizaje; supervisión; enseñanza clínica 


\section{Introdução}

O contexto clínico é um espaço de aprendizagem de relevo para a formação profissional do enfermeiro, caraterizando-se pela transformação do conhecimento com recurso a métodos e estratégias dinâmicas e interativas como a observação, a reflexão, debate e análise. Rua (1) infere que o ensino clínico é "um período importante para formação do aluno como forma de desenvolvimento de capacidades, competências e atitudes profissionais que não são suscetíveis de desenvolvimento em contexto virtual de laboratório".

Tendo por base estes pressupostos, os conhecimentos e saberes apropriados em período teórico são transformados em contexto da prática profissional, não alienando os pressupostos atuais da formação de enfermagem de excelência em que a aprendizagem centrada no estudante, integra o contexto e a organização $(2,3)$.

Face aos novos desafios dos processos de formação em enfermagem, emerge a importância da supervisão que tem sido foco de atenção quer nas instituições de formação quer nas de saúde em Portugal.

A supervisão é "caraterizada por ser um processo de acompanhamento de uma atividade e da ou das pessoas que a realizam orientado no sentido de facilitar a boa consecução da atividade o desenvolvimento da competência e o grau de satisfação de quem a executa" (3). Deste modo, trata-se de um instrumento transformador, de um processo dinâmico, interpessoal, interativo, ecológico, mediador e facilitador, sustentado numa lógica de relação entre os vários atores, com o objetivo de desenvolver competências tendo como propósito um ensino de enfermagem de qualidade.

O modelo de supervisão de estudantes vigente no contexto da nossa pesquisa preconiza a existência de uma equipa constituída pelo gestor pedagógico, docente que desempenha funções integrantes e integradoras do processo de ensino e de aprendizagem teórico e prático, responsável pelo processo formativo dos estudantes antes, durante e após o ensino clínico; enfermeiro tutor, enfermeiro que exerce funções no contexto clínico e que tem uma participação direta e ativa na aprendizagem do estudante observando, ajudando no seu desempenho, tem uma relação mais próxima do gestor pedagógico e a sua intervenção tem como unidade de tempo o período de duração do ensino clínico; e enfermeiro de referência, o enfermeiro prestador de cuidados do contexto de trabalho que participa na orientação de forma esporádica, tendo por unidade de tempo o turno de trabalho (4).

À luz da importância e relevância do processo de supervisão no desenvolvimento da aprendizagem e das competências dos estudantes de enfermagem, desenvolvemos um estudo com os seguintes objetivos: conhecer a perceção dos enfermeiros tutores sobre a supervisão de estudantes de enfermagem em ensino clínico, e conhecer a perceção dos estudantes de enfermagem sobre a supervisão em ensino clínico realizada pelos enfermeiros tutores, com a finalidade de contribuir com propostas para a praxis da supervisão.

Salienta-se que os resultados apresentados no artigo se referem à pesquisa efetuada no ano de 2012 e que integram um estudo mais amplo, que iniciou em 2011 com término previsto em 2015. 


\section{Metodo}

Tendo em conta os objetivos do estudo, optámos pelo paradigma qualitativo, descritivo-exploratório. Atendendo à metodologia, objetivos e finalidade do estudo optámos pela entrevista semiestruturada como instrumento de recolha de dados. Foi construído um guião com questões abertas para cada grupo de participantes e realizado pré-teste. Para os estudantes de enfermagem o guião final foi composto por duas partes, a primeira para a caraterização sociodemográfica e a segunda integrando as seguintes questões: o que entende por supervisão? O que valoriza mais relativamente ao processo de supervisão?; Como se tem sentindo neste processo de supervisão?, e que sugestões daria para a melhoria deste processo?

Para os enfermeiros tutores foi seguida a mesma metodologia e a primeira parte da entrevista permitiu a caraterização sociodemográfica e profissional e a segunda parte foi composta pelas seguintes questões: O que significa para si a supervisão de estudantes de enfermagem? Que estratégias utiliza neste processo? Que dificuldades/necessidades sente na supervisão dos estudantes de enfermagem? Que sugestões daria para a melhoria deste processo?

O estudo foi realizado numa Escola Superior de Enfermagem da zona norte de Portugal e os participantes foram selecionados de acordo com os seguintes critérios: estudantes a frequentarem o curso de licenciatura em enfermagem que tenham realizado pelo menos um ensino clínico e enfermeiros tutores supervisores, dos estudantes que participam no estudo.

A recolha de dados foi realizada em julho de 2012, por três dos cinco investigadores, que recorreram à gravação áudio e procederam à sua transcrição.

O tratamento dos dados efetuou-se com recurso a técnicas de análise de conteúdo, segundo Bardin (5), tendo sido respeitados todos os procedimentos ético-deontológicos inerentes à realização de um trabalho de investigação, segunda a Declaração de Helsínquia de 2013.

Neste sentido, analisámos a informação obtida seguindo as etapas preconizadas por Bardin (5): pré-análise; exploração do material e tratamento dos resultados. Constituído o corpus de análise, foi fragmentado em unidades de registo procedendo-se à sua categorização permeada pela exaustividade, homogeneidade, pertinência, objetividade, fidelidade e produtividade. Este processo foi realizado pelos outros dois investigadores e posteriormente aferida por todos.

\section{Apresentação e discussão dos resultados da investigação}

O processo de supervisão, como ferramenta fundamental para a aprendizagem no ensino clínico, integra dinâmicas e atividades de orientação e acompanhamento. Corresponsabilizar os estudantes e supervisores na condução do percurso formativo é fundamental e para isso, é necessário conhecer as suas perceções sobre o modelo de supervisão em uso.

Neste estudo participaram quinze estudantes de enfermagem e seis enfermeiros tutores. Do grupo de estudantes, dez frequentavam o $2^{\circ}$ ano do curso e cinco o $3^{\circ}$ ano, sendo doze do sexo feminino e com média de idade vinte e dois anos. Os enfermeiros tutores são, maioritariamente do sexo 
feminino (cinco), idades compreendidas entre os 37 e os 57 anos, com média de idades de 43 anos. Têm em média 21 anos de serviço, 13 anos como supervisor de estudantes de enfermagem e 2 possuem formação específica na área de supervisão.

\section{Perspectiva dos estudantes}

Analisados os discursos dos estudantes, emergiram as seguintes áreas temáticas: conceito de supervisão; perceção relativa ao processo de supervisão; estratégias de supervisão mobilizadas pelos tutores; impacto do processo de supervisão e sugestões de melhoria do processo de supervisão. Em cada área temática surgiram várias categorias conforme se pode analisar na figura 1.

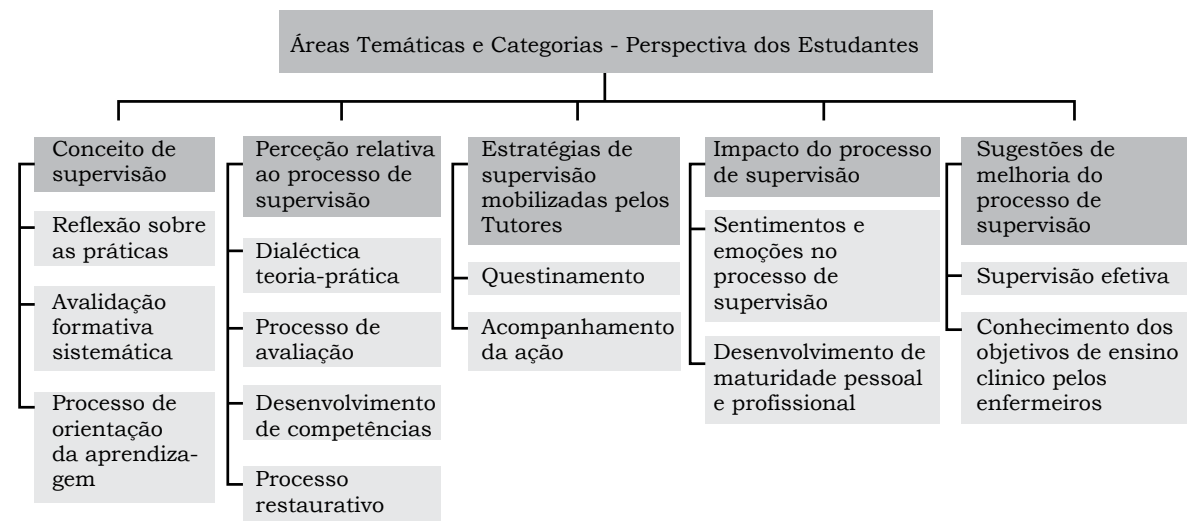

Figura 1. Áreas temáticas e categorias: perspectiva dos estudantes

FonTE: feito pelos autores

Segundo os estudantes de enfermagem o conceito de supervisão é entendido como a reflexão sobre as práticas, a avaliação formativa sistemática e um processo de orientação da aprendizagem. Esta conceção corrobora a perspectiva de diferentes autores encontrados na revisão da literatura, onde na essência do processo de supervisão se encontra o acompanhamento das atividades, processos de regulação e mediação da aprendizagem, monitorização das ações e a avaliação (1-3,6).

Quanto ao processo de orientação da aprendizagem os estudantes entendem existir um acompanhamento sistemático, favorecedor da evolução da aprendizagem e facilitador na deteção e correção de incidentes críticos, como exemplificado na unidade de registo "é o acompanhamento... de nos fazer pensar, de refletir o porquê de algo... De nos incentivar a pesquisar, a saber mais e a alimentar o nosso conhecimento [...]" (EE12). Neste sentido, a supervisão é concebida como formativa e potenciadora das aprendizagens, no entanto, assume também uma natureza de controlo e de mediação, tal como é mencionado por Alarcão e Canha (3). Abreu $(6,7)$ acrescenta que esta 
mediação integra identidades individuais e coletivas, das quais resultam três componentes: cognitiva, avaliativa e emocional. Para os estudantes este processo integra uma transformação de saberes através dos quais determinada experiência se eleva a uma compreensão mais consubstancial onde se interliga o saber do contexto (6), constituindo a aprendizagem significativa.

No que diz respeito à perceção relativa ao processo de supervisão, percebem-no como um processo que permite o desenvolvimento de competências do estudante e do supervisor. Permite a dialética teoria-prática, pelo reconhecimento dos mesmos princípios de orientação "[...] estão no mesmo... no mesmo lado, entre aspas que as enfermeiras orientadoras. Ou seja, aquilo que nós [...] que é preconizado na escola, também penso que está a ser seguido pelas enfermeiras tutoras" (EE1), e continuidade no processo de aprendizagem, corroborado por Saarikoski et al. (8) que num estudo que realizaram constataram da importância do supervisor no favorecimento desta perspectiva da articulação teoria-prática.

Os estudantes percebem ainda, que a supervisão integra o processo de avaliação, realçando, a avaliação na sua dimensão formativa quando realizada de forma sistemática, tal como se verifica no depoimento de um dos estudantes "[...] o que realmente valorizo mais é o facto deles estarem preocupados em conhecer as nossas dificuldades e os nossos constrangimentos e ajudar-nos para que as nossas próximas experiências sejam cada vez melhores e mais gratificantes e possamos crescer com isso" (EE6). Osborn e Kelly (9) consideram que a modalidade de avaliação formativa é uma atividade do supervisor e da supervisão no sentido da promoção do processo de supervisão, de aprendizagem e desenvolvimento de competências.

Este último aspeto constitui uma das perceções que referem os estudantes de enfermagem em relação ao processo de supervisão. Estas competências são apreendidas sob forma pessoal de aprendizagem e orientação e integram processos que alicerçam o ensino clínico, tais como: racionais, analíticos, empíricos e metafóricos (6).

Os estudantes percebem, ainda, a supervisão como um processo de desenvolvimento de competência nas diferentes vertentes, para os quais se pressupõe o estabelecimento de uma relação supervisiva de abertura e confiança, capaz de se traduzir em apoio, evidenciando a sua função restaurativa $(3,6,10)$ categorizada neste estudo como processo restaurativo, como podemos constatar na voz dos estudantes "A empatia, a relação que neste estágio (a tutora) criou connosco, deixou-nos completamente à vontade para questionar" (EE12). Para isso, torna-se premente que esta supervisão se foque na formação integral do estudante, onde a sua aprendizagem se centre nos valores do ser pessoa e não no estatuto que lhe confere, tal como entendido por Gomez (11).

As Estratégias de supervisão mobilizadas pelos tutores no ensino clinico assentam no questionamento, como expressa a unidade de registo "Normalmente eles vão perguntando, por exemplo quando temos um doente eles vão perguntando o diagnóstico, depois vão tentando relacionar a medicação que o doente toma com o diagnóstico e porquê [...]" (EE1) e no acompanhamento da ação: "Nos primeiros dias, nas primeiras semanas e mesmo 
agora que estamos a meio do ensino clínico, ainda é feito... esse acompanhamento que na minha opinião é muito importante" (EE2).

Estas estratégias configuram-se como promotoras da reflexão e fomentam a coresponsabilização dos atores com impacto no desenvolvimento das aprendizagens, indo de encontro aos objetivos preconizados para o ensino clínico, tal como, verificamos nos estudos de Kristofferzon et al. (10) e Löfmark et al. (12).

O Impacto do processo de supervisão relaciona-se com o desenvolvimento de sentimentos e emoções no processo de aprendizagem e desenvolvimento de maturidade pessoal e profissional, como, plasmados nos seguintes depoimentos: "[...] sentimos sempre um bocadinho de receio, com medo... mas isso é necessário ao nosso processo de evolução" (EE2); “[...] acho que existe muita pressão... temos muito medo de errar e acho que os nervos também não ajudam [...]" (EE5); "os alunos enquanto alunos de enfermagem necessitam dessa orientação para poderem crescer como pessoas e como futuros profissionais de saúde" (EE6).

Hellström-Hyson et al. (13) realizaram um estudo onde concluíram que a supervisão permite o desenvolvimento de autoconfiança, responsabilização, a capacidade para o juízo clínico e a maturidade, estando estes resultados em consonância com os do nosso estudo.

Os estudantes de enfermagem dão como Sugestões de melhoria do processo de supervisão: uma supervisão efetiva e conhecimento dos objetivos do ensino clínico pelos enfermeiros, das unidades de saúde, onde decorrem os mesmos. Também, SaariKoski et al. (8) no seu estudo realçam que muitos dos enfermeiros desconhecem os desenvolvimentos curriculares dos ensinos clínicos. Consideramos que os aspetos referidos pelos estudantes carecem de uma intervenção por partes das instituições parceiras (escola e unidades de saúde) de forma a minimizar este impacto no processo de aprendizagem dos estudantes em ensino clínico.

\section{Perspectiva dos enfermeiros tutores}

Da análise dos discursos dos enfermeiros tutores interessa realçar que as áreas temáticas: conceito de supervisão; estratégias de supervisão mobilizadas e perceção relativa ao processo de supervisão são coincidentes com os achados dos discursos dos estudantes. Sobressaíram, ainda, dificuldades e necessidades vividas na supervisão e sugestões relativas ao processo de supervisão dos estudantes.

Em todas as áreas temáticas emergiram várias categorias que a seguir se apresentam na figura 2. 


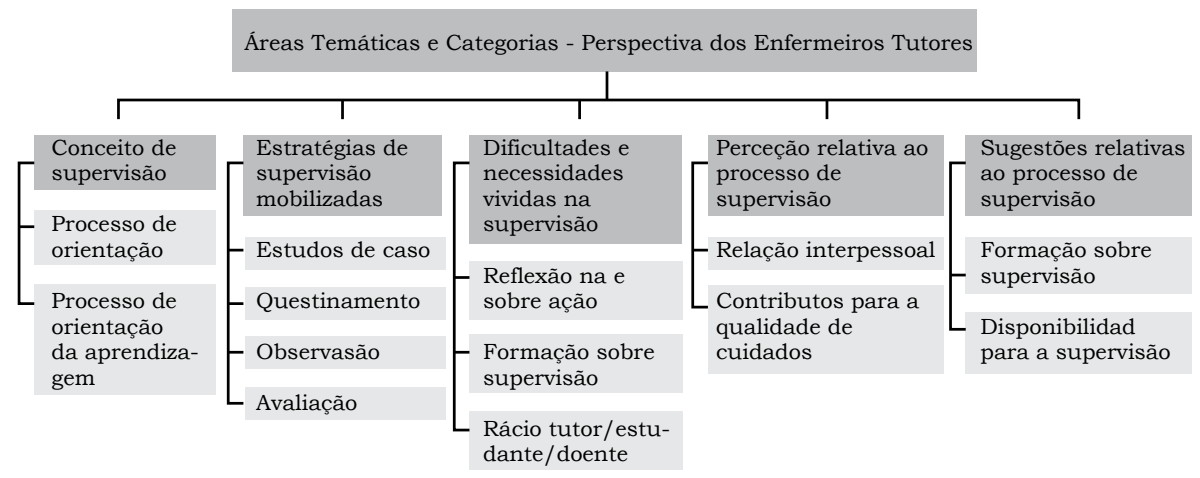

Figura 2. Áreas temáticas e categorias: perspectiva dos enfermeiros tutores

FonTE: feito pelos autores

O conceito de supervisão que emergiu das falas dos enfermeiros tutores relaciona-se com um processo de orientação e processo facilitador $d a$ aprendizagem, tal como sugere a unidade de registo "[...] A supervisão é um processo facilitador da mobilização e intervenção de conhecimentos teóricos na prática" (ET5).

Numa análise conjunta pudemos constatar que também os estudantes nos seus depoimentos concebem supervisão como um processo de orientação e potenciador da aprendizagem pela reflexão sobre as práticas. A mesma abordagem é evidenciada por Alarcão e Canha (3) quando a caracterizam como um processo de acompanhamento orientado no sentido de promover as atividades cujo fim último é o desenvolvimento de competência e a satisfação de quem as executa.

As Estratégias de supervisão mobilizadas contemplam: realização de estudos de caso, questionamento "[...] é muito importante este constante questionamento do aluno" (ET2), observação "após essa observação faço muitas questões acerca do doente, da patologia, da medicação" (ET2) e avaliação.

Todas estas estratégias são mencionadas em diferentes estudos, no entanto destacamos a observação pela sua representação interativa, uma vez que "o olhar e a capacidade de visão são elementos fundamentais no processo de acompanhamento supervisivo" (3). Mais uma vez verificamos que as estratégias identificadas pelos estudantes de enfermagem são coerentes com as dos enfermeiros tutores. No entanto, estes dão enfase a outras estratégias estimuladoras e promotoras da reflexão na ação. É interessante constatar que os estudantes percecionam a avaliação integrando o conceito de supervisão mas, não enquanto estratégia. Neste contexto, a revisão de literatura aponta neste sentido, salientado como estratégias de supervisão, análises de caso, perguntas pedagógicas e avaliação na sua modalidade formativa entre outras $(3,6,9,14)$. 
Da análise das Dificuldades e necessidades vividas na supervisão, evidenciadas pelos enfermeiros tutores emerge a: reflexão na e sobre a ação, formação sobre supervisão e rácio tutor/estudante/doente.

A acumulação de funções de supervisão com a de prestação de cuidados pelos enfermeiros tutores "[...] ao mesmo tempo estou com doentes a prestar cuidados e é muito difícil" (ET3), também, refletido pelo número elevado de estudantes e utentes, confere pouca disponibilidade para a reflexão e para a otimização do processo. Assim, parece-nos poder dizer que a sobrecarga de trabalho destaca-se como uma das dificuldades no processo de supervisão, tal como concluíram Jiménez et al. (15).

A formação sobre supervisão emerge como necessidade: "Acho que era importante todos nós termos formação nesta área, acho que era fundamental" (ET5), também, corroborado no estudo de Fernandes et al. (16) os quais concluíram que a formação se faz sentir em diferentes áreas, nomeadamente: técnica, relacional e pedagógica, de modo a que estes atores fiquem mais aptos no desempenho das suas funções como supervisores.

Estes resultados podem explicar a sugestão manifestada pelos estudantes sobre a necessidade de uma supervisão efetiva a realizar pelos enfermeiros tutores. Numa análise das sugestões relativas ao processo de supervisão relatadas pelos enfermeiros tutores: formação sobre supervisão e disponibilidade para a supervisão, percebe-se que emergem no sentido de dar resposta e colmatar as dificuldades e necessidades por eles sentidas.

Na perceção relativa ao processo de supervisão foram evidenciadas a relação interpessoal e contributos para a qualidade de cuidados. Os enfermeiros tutores percebem e entendem o processo de supervisão nas suas dimensões formativa e relacional com a intencionalidade de potenciar a aprendizagem e o desenvolvimento de competências, tal como preconizam diversos autores $(2,3,6,17)$ e como temos vindo a enfatizar.

Em síntese, os estudantes participantes neste estudo, consideram a supervisão em ensinos clínicos de enfermagem como um processo de acompanhamento das suas práticas e de orientação. Realçam que esta perspectiva de supervisão é facilitadora do desenvolvimento da maturidade pessoal e profissional e desencadeadora de sentimentos e emoções no processo aprendizagem, permitindo o desenvolvimento de competências dos supervisores e supervisados.

Os enfermeiros tutores entendem a supervisão em ensinos clínicos de forma análoga, complementando-a com a dimensão relacional do processo e em consonância com Abreu (7) para quem a supervisão é um "processo dinâmico, interpessoal e formal de suporte, acompanhamento e desenvolvimento de competências profissionais, através da reflexão, ajuda, orientação e monitorização, tendo em vista a qualidade dos cuidados de enfermagem". As condições de trabalho dos contextos da prática parecem ser os elementos mais condicionadores do processo de supervisão de estudantes, embora também refiram necessidade de realizarem formação específica nesta área.

À luz dos achados, articulando-os com o quadro referencial teórico, a supervisão em enfermagem reflete-se no desenvolvimento de competências terapêuticas e relacionais dos estudantes, tornando-se uma ferramenta de 
alta qualidade e imprescindivel no desenvolvimento de futuros enfermeiros e da enfermagem. De acordo com Abreu (6), "a promoção da qualidade é o resultado da construção de uma prática mais racional, estruturada, integrada, e funcional, baseada na melhoria dos processos, aprofundamento das estratégias e reforço da relação entre a comunidade educativa e organização". Nesta perspectiva a supervisão em enfermagem deve ter por base quadros referenciais que alicercem a mudança e o processo de construção social da profissão.

\section{Conclusão}

Dos resultados deste estudo podemos inferir que, tanto os estudantes de enfermagem como os enfermeiros tutores, possuem perceções análogas da supervisão em ensinos clínicos de enfermagem, cuja primeira intenção passa por potenciar a aprendizagem e o desenvolvimento de competências pessoais e profissionais.

Os estudantes dão enfase ao processo de supervisão efetiva como arma terapêutica primordial para o desenvolvimento de habilidades e competências e para um percurso de aprendizagem sustentado, que conduz ao desenvolvimento pessoal.

Os enfermeiros tutores apontam dificuldades/necessidades que se inserem na dimensão formativa, particularmente no défice de formação sobre supervisão, e ainda, dificuldades que se prendem com acumulação de papéis. No sentido de minimizar esta diversidade de funções dos enfermeiros tutores e contribuir para uma melhoria da qualidade dos processos formativos e da qualidade dos cuidados, é necessário uma maior aposta quer na formação, quer na sensibilização dos gestores dos contextos clínicos face às funções desempenhadas por estes enfermeiros tutores.

\section{Conflito de interesses}

Não existe qualquer conflito de interesses dos autores em relação a este estudo.

\section{Financiamento}

Não houve apoio financeiro.

\section{Referências}

1. Rua M. De aluno a enfermeiro, desenvolvimento de competências em contexto de ensino clínico. Loures: Lusociência; 2011.

2. Fonseca MJL. Supervisão em ensinos clínicos de enfermagem. Coimbra: Formasau; 2006.

3. Alarcão I, Canha B. Supervisão e colaboração: uma relação para o desenvolvimento. Porto: Porto Editora; 2013.

4. Escola Superior de Saúde. Modelos, modos e modalidade de formação. Viana do Castelo: Instituto Politécnico de Viana do Castelo; 2000.

5. Bardin L. Análise de conteúdo. Lisboa: Edições 70; 2011.

6. Abreu W. Formação e aprendizagem em contexto clínico. Coimbra: Formasau; 2007. 
7. Abreu W. Supervisão, qualidade e ensinos clinicos: que parcerias para a excelência em saúde? Coimbra: Formasau; 2003.

8. Saarikoski M, Warne T, Päivi K, Leino-Kilpi H. The role of nurse teacher in clinical practice: an empirical study of Finnish student nurse experiences. Nurse Educ Today [internet]. 2009 [citado 2014 mar 2];29(6):595-600. Disponivel em: http://www.sciencedirect.com/ science/article/pii/S0260691709000173

9. Osborn CJ, Kelly BL. No Surprise: practices for conducting supervisee evaluations. En: Culbreth JR, Brown LL. State of the art in Clinical Supervision. New York: Routledge; 2010.

10. Kristofferzon ML et al. Nursing students perceptions of clinical supervision: The contributions of preceptors, had preceptors and clinical lecturers. Nurse Educ Today [internet]. 2012 [citado 2013 abri 30];33(10):1252-7. http://dx.doi.org/10.1016/j.nedt.2012.08.017

11. Gómez AG. La formación integral de estudiantes de enfermería. Investig Enferm. Imagen Desarr [internet]. 2013 jul-dez [citado 2015 abr 4];15(2):153-72. Disponivel em: http://www.redalyc.org/ pdf/1452/145229803009.pdf

12. Löfmar A, Thorkildsen K, Räholm MB, Natvig GK. Nursing student's satisfaction whith supervision from preceptors and teachers during clinical practice. Nurse Educ Pract [Internet]. 2012 [citado 2013 abr 30];12(3):164-9. Disponível em: http://www.elsevier.com/nepr

13. Hellström-Hyson E, Martensson G, Kristofferzon ML. To take responsibility or to be onlooker. Nursing students' experiences for two models of supervision. Nurse Educ Today [internet]. 2012 jan. [citado 2014 abr 1];32(1):105-10. Disponível em: http://www.sciencedirect. com/science/article/pii/S0260691711000487

14. Alarcão I, Tavares J. Supervisão da prática pedagógica: uma perspectiva de desenvolvimento e aprendizagem. $2^{\text {a }}$ ed. Coimbra: Almedina; 2003.

15. Jiménez GA, Herrera FEL, Núnera DMR. La experiencia educativa del profesional de enfermeria en el ámbito clínico. Investig Enferm. Imagen y Desarr [internet]. 2013 Jul-dez [citado 2015 mar 9];15(2):9-29. Disponivel em: http://www.redalyc.org/pdf/1452/145229803002.pdf

16. Fernandes D, Borralho A, Vale I, Mendonça V, Gaspar A, Fidalgo C. Uma avaliação dos processos de aprendizagem: ensino e avaliação numa Escola Superior de Enfermagem [internet]. Lisboa: Instituto de Educação da Universidade de Lisboa; 2012 [citado 2015 mar 17]. Disponivel em: http://hdl.handle.net/10451/10916

17. Garrido A, Simões J, Pires R. Supervisão clínica em enfermagem perspectivas práticas. Aveiro: Universidade de Aveiro; 2008. 